\title{
Light-Induced Rescue of Breathing after Spinal Cord Injury
}

\author{
Warren J. Alilain, ${ }^{1}$ Xiang Li, ${ }^{1}$ Kevin P. Horn, ${ }^{1}$ Rishi Dhingra, ${ }^{2}$ Thomas E. Dick,,${ }^{1,2}$ Stefan Herlitze, ${ }^{1}$ and Jerry Silver ${ }^{1}$ \\ ${ }^{1}$ Department of Neurosciences and ${ }^{2}$ Division of Pulmonary, Critical Care, and Sleep Medicine, Department of Medicine, Case Western Reserve University \\ School of Medicine, Cleveland, Ohio 44106
}

\begin{abstract}
Paralysis is a major consequence of spinal cord injury (SCI). After cervical SCI, respiratory deficits can result through interruption of descending presynaptic inputs to respiratory motor neurons in the spinal cord. Expression of channelrhodopsin-2 (ChR2) and photostimulation in neurons affects neuronal excitability and produces action potentials without any kind of presynaptic inputs. We hypothesized that after transducing spinal neurons in and around the phrenic motor pool to express ChR2, photostimulation would restore respiratory motor function in cervical SCI adult animals. Here we show that light activation of ChR2-expressing animals was sufficient to bring about recovery of respiratory diaphragmatic motor activity. Furthermore, robust rhythmic activity persisted long after photostimulation had ceased. This recovery was accomplished through a form of respiratory plasticity and spinal adaptation which is NMDA receptor dependent. These data suggest a novel, minimally invasive therapeutic avenue to exercise denervated circuitry and/or restore motor function after SCI.
\end{abstract}

Key words: spinal cord injury; paralysis; respiration; motor neuron; plasticity; NMDA receptor; kindling

\section{Introduction}

Expression of the algal protein Channelrhodopsin-2, a rapid and light-activated cation channel, in mammalian neurons via viral gene delivery can manipulate neuronal spiking and create action potentials after light exposure in vitro (Boyden et al., 2005; Li et al., 2005; Zhang et al., 2006, 2007a; Herlitze and Landmesser, 2007). Recent studies have demonstrated that the swimming behavior of nematodes can be influenced by light activation of ionic channels and that these light sensitive channels can be expressed in living mammalian CNS tissue, where they can drive useful and functional activity within neuronal circuits (Li et al., 2005; Arenkiel et al., 2007; Zhang et al., 2007b; Huber et al., 2008; Lagali et al., 2008).

One potential and powerful application of these dynamic light switches is in the treatment of neurological diseases and traumatic CNS injuries, in particular spinal cord injury (SCI). The disruption of descending inputs to motor neurons after SCI results in loss of motor function. It is the interruption of presynaptic inputs to motor neurons after SCI that makes it an ideal disorder model to use the ChR2 light switch and to activate these otherwise quiescent or dormant neurons because regeneration of severed axons to reinnervate target neurons and restore function is, as of now, not yet a viable therapy (Houle et al., 2006). In our

Received July 18, 2008; revised Sept. 10, 2008; accepted Sept. 14, 2008.

This work was supported by The Christopher and Dana Reeve Foundation (W.J.A.), National Institute of Neurological Disorders and Stroke (NINDS) Grants NS25713 and NS060767 (J.S.), the Brumagin Memorial Fund (J.S.), NINDS Grant NS047752 (S.H.), and National Heart, Lung, and Blood Institute Grant HL080318 (T.E.D.). We also acknowledge Drs. Lynn Landmesser and Richard Sidman and Ermelinda Ceco for their insightful comments on this manuscript and Hongmei Hu for her technical assistance.

Correspondence should be addressed to Dr. Jerry Silver, Department of Neurosciences, Case Western Reserve University School of Medicine, Room E-658, 2109 Adelbert Road, Cleveland, 0H 44106. E-mail: jxs10@case.edu.

DOI:10.1523/JNEUROSCI.3378-08.2008

Copyright $\odot 2008$ Society for Neuroscience ～0270-6474/08/2811862-09\$15.00/0 present experiments we used the C2 hemisection model of SCI on adult female Sprague Dawley rats.

Injuries at the cervical level are one of the most common types of SCI and often result in respiratory insufficiency (National Spinal Cord Injury Statistical Center, 2006). In the C2 hemisection model, there is an interruption of the descending bulbospinal inputs to the ipsilateral phrenic nucleus, which innervates the hemidiaphragm, resulting in unilateral paralysis (Fig. 1A) (Goshgarian, 2003; Zimmer et al., 2007). Electromyographic (EMG) activity can be partially restored to the paralyzed hemidiaphragm through activation of an ineffective, latent pathway that arises from premotor neurons in the ventrolateral respiratory column and whose axons descend contralateral to the $\mathrm{C} 2$ hemisection and cross over caudal to the lesion to innervate phrenic motor neurons (PMNs) (Fig. 1A) (Goshgarian, 2003; Zimmer et al., 2007). However, spontaneous activation of this so-called "crossed phrenic pathway" is slow to develop and interventional processes to activate it can be stressful to the animal, i.e., contralateral phrenicotomy leading to asphyxiation or intermittent hypoxia (Porter, 1895; Nantwi et al., 1999; Fuller et al., 2003; Goshgarian, 2003; Golder and Mitchell, 2005; Zimmer et al., 2007).

An important advantage of ChR2 technology is that it is a relatively noninvasive technique capable of powerfully stimulating CNS circuit activity (Zhang and Oertner, 2007; Zhang et al., 2008). We tested the hypothesis that after C2 hemisection and infection of spinal neurons at the level of the phrenic nucleus to express ChR2, patterned photostimulation would lead to a recovery of motor function and a return of hemidiaphragmatic activity through direct or indirect stimulation of phrenic motor neurons or potentiation of the phrenic nucleus to spared inputs.

\section{Materials and Methods}

C2 hemisection and virus injection. Adult female Sprague Dawley rats (250-300 g) were anesthetized with a ketamine $(70 \mathrm{mg} / \mathrm{kg})$ and xylazine 

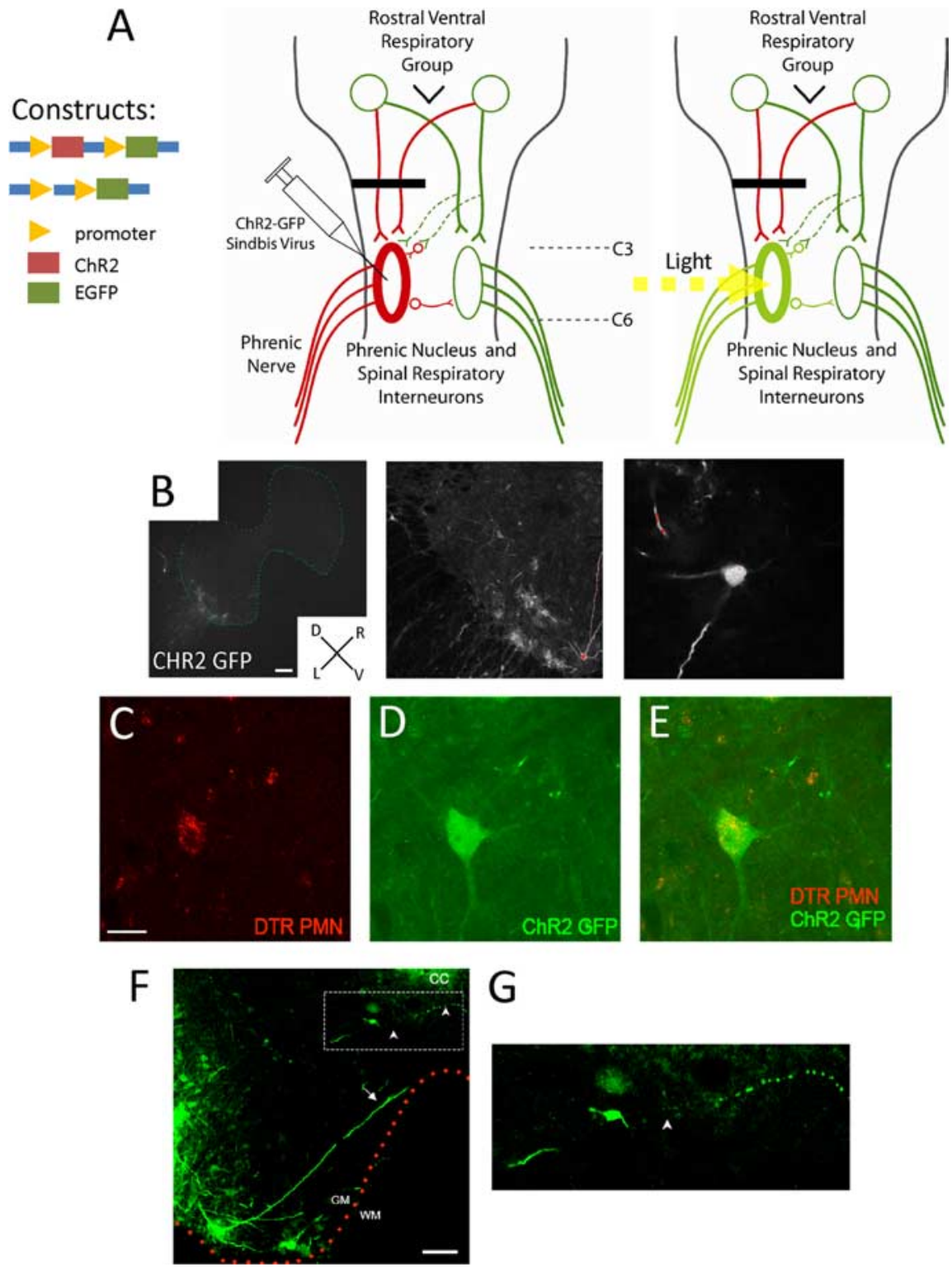

Figure 1. Expression of ChR2-GFP in cervical spinal cord neurons after injection of a Sindbis virus into $(2$ hemisected animals. $A$, Schematic of the (2 hemisection (black line), crossed phrenic pathway (dashed green lines), and ChR2-GFP photostimulation treatment protocol. After $\mathrm{C} 2$ hemisection, bulbospinal inputs to the ipsilateral phrenic nucleus are interrupted resulting in a quiescent phrenic nerve (red lines) and paralysis of the ipsilateral hemidiaphragm. At the same time of lesioning, ipsilateral C3-C6 spinal neurons, including contralateral projecting interneurons, are infected with a Sindbis virus to express ChR2 and GFP. After $4 \mathrm{~d}$, the $\mathrm{C3}-\mathrm{C} 6$ spinal cord is exposed to light to stimulate the phrenic nerve and reactivate the paralyzed ipsilateral hemidiaphragm. $\boldsymbol{B}$, Treatment with Sindbis virus containing ChR2-GFP leads to GFP expression in ipsilateral C3-C6 spinal neurons. In addition, treatment with ChR2-GFP Sindbis virus leads to GFP expression in C3-C6 phrenic motor neurons retrogradely labeled with Dextran Texas Red. D, Dorsal; V, ventral; L, left; R, right. Scale bar, $200 \mu \mathrm{m}$. C, Dextran Texas Red-labeled phrenic motor neuron. Scale bar, $50 \mu \mathrm{m}$. D, GFP expression of Sindbis virus containing ChR2-GFP. $\boldsymbol{E}$, Overlay of Dextran Texas Red-labeled phrenic motor neurons expressing GFP. F, Both interneurons and motor neurons infected with ChR2-GFP send neurites across or toward the midline and are in a position to potentially affect contralateral neurons and/or motor output. Arrows point to motor neuronal neurites projecting to the midline, and arrowheads point to interneuronal neurites. Scale bar, $100 \mu \mathrm{m}$. G, Enlarged image (dotted line rectangle) of interneurons with midline projecting neurites.

( $7 \mathrm{mg} / \mathrm{kg}$ ) solution administered intraperitoneally. After administration of the anesthetic mixture, the animals were prepared for surgery by shaving and cleansing the dorsal neck area with betadine and 70\% alcohol. After the surgical prep, about a $4 \mathrm{~cm}$ midline incision was made on the neck. After retraction of the paravertebral muscles, a multilevel laminectomy was performed and the dura and arachnoid mater were cut with microscissors to expose several cervical segments of the animal's spinal cord. A left C2 hemisection just caudal to the C2 dorsal root was made with a sharp microblade. The hemisection was made from the midline to the lateral most extent of the spinal cord.

At the same time as hemisection, the animals received three injections of Sindbis virus $(250 \mathrm{nl}$ per injection) containing either the dual ChR2GFP vector $(n=14)$ or the green fluorescent protein (GFP) vector alone $(n=9)$ into the C3-C6 region of the spinal cord, the level of the phrenic motor nucleus. Injections were made ipsilateral to the lesion, $0.11 \mathrm{~cm}$ from the midline and $0.16 \mathrm{~cm}$ ventral from the dorsal surface of the spinal cord, in close proximity to the phrenic nucleus, through use of a Kopf stereotaxic device. Sham/nonlesioned animals received all procedures but the hemisection $(n=$ 10). Of these 10, six received the ChR2-GFP construct, and four received control injection.

Following these procedures, the paravertebral muscles were sutured back together with 3-0 vicryl and the skin stapled together with wound clips. Animals received marcaine and buprenorphine for analgesia. Saline was administered subcutaneously if the animals appeared dehydrated. The animals were housed in normal day/night schedule and given food and water ad libitum.

Constructs and virus. Sindbis virus vector $\operatorname{SinRep}\left(n s 2^{2} \mathrm{~S}^{726}\right)$ and helper DH-BB were kindly provided by P. Osten (Northwestern University, Evanston, IL) (Kim et al., 2004). $\operatorname{SinRep}\left(n s P 2 S^{726}\right)$ dSP-EGFP was constructed by subcloning another subgenomic promoter with EGFP into the ApaI site of the original $\operatorname{SinRep}\left(n s P 2 S^{726}\right)$. cDNA of ChR2 (GenBank accession no. AF461397) was PCR-amplified and cloned into the $\mathrm{XbaI}$ and MluI sites of $\operatorname{SinRep}\left(n s P 2 S^{726}\right)$ dSP-EGFP under a CMV promoter. Sindbis pseudovirions were prepared according to Invitrogen's directions (Sindbis Expression System) and then concentrated with an ultracentrifuge. Viral titer was $0.5-1 \times 10^{8}$ units per $\mathrm{ml}$.

EMG recordings and light stimulation. Four days after $\mathrm{C} 2$ hemisection and/or virus injection the animals were anesthetized as above and prepared for light treatment and physiological recordings. In a room where all light was eliminated except for that needed to accomplish the surgical procedures, approximately an eight $\mathrm{cm}$ incision was made at the base of the ribcage to expose the abdominal surface of the diaphragm. Bipolar electrodes, connected to an amplifier and data acquisition set-up (CED 1401/Spike2 Data Analysis Computer Interface, Cambridge Electronic Design), were inserted into both left and right hemidiaphragms to record diaphragmatic activity. After this, the cervical area of the spinal cord was reopened again for exposure to photostimulation at a wavelength of $475 \mathrm{~nm}$, i.e., blue light. The light source was a portable unit capable of producing light at various wavelengths through a fiber optic cable (Model Lambda DG-4, Sutter Instrument). Diaphragmatic motor activity was recorded before, during, and after stimulation. During recording, the animals were placed on a circulating warm water blanket to maintain body temperature. The initial protocol used for photostimulation included sustained exposure (1 min) of the C3-C6 spinal cord from the light source, as well as, intermittent exposure to light at about once per second for $1 \mathrm{~min}$. In 
animals that received the longer lasting light delivery protocol which resulted in more robust recovery $(>1 \mathrm{~h})$, the following light stimulation protocol was used: alternating $5 \mathrm{~min}$ rest $/ 5 \mathrm{~min}$, intermittent light stimulation for three or four cycles $(30-40 \mathrm{~min}$ total). This protocol was adapted from the long-term facilitation induction protocol of $5 \mathrm{~min}$ normoxia followed by 5 min hypoxia (Fuller et al., 2003; Golder and Mitchell, 2005). The intermittent stimulation consisted of light exposure at $0.5 \mathrm{~Hz}$, with each flash of light $1 \mathrm{~s}$ long.

NMDA receptor blockade with MK-801. To block NMDA receptors, $500 \mu \mathrm{l}$ of $10 \mu \mathrm{M}$ MK-801 (Sigma), a noncompetitive NMDA receptor antagonist, diluted in PBS, was applied to the exposed spinal cord. MK801 was administered after 5 min of baseline recording. Recording with MK-801 continued for 5 more minutes before intermittent photostimulation and thereafter as described above.

Data and statistical analysis. After recording, the raw diaphragmatic EMG signal was rectified and integrated using Spike2 software. Frequency was determined by counting total breaths for 5 min before and after photostimulation. Peak amplitude and burst duration of inspiratory bursts were measured through Spike2, for at least 25 breaths before and after stimulation. Poststimulation analyses of ChR2 animals were made during regular patterned respiratory related diaphragmatic EMG activity. All values were standardized to prestimulation measures. Statistical analysis was performed using ANOVA and Tukey's post hoc analysis. All values with a $p$ value $<0.05$ were considered significant. All error bars indicate SEs.

Fluorescence and immunocytochemistry analysis. For immunocytochemical experiments, phrenic motor neurons were retrogradely labeled with Dextran Texas Red (Invitrogen) at the time of hemisection. Animals were anesthetized as above and about an $8 \mathrm{~cm}$ incision was made at the base of the ribcage to expose the abdominal surface of the diaphragm. Five $10 \mu \mathrm{l}$ aliquots of $0.4 \%$ Dextran Texas Red were injected into the left hemidiaphragm. The abdominal muscles were sutured together and the skin stapled together with wound clips.

Four days after injection of tracer or immediately after recording, animals were perfused first with $50 \mathrm{ml}$ of PBS, followed by $250 \mathrm{ml}$ of chilled $4 \%$ paraformaldehyde in PBS. The cervical spinal cord was harvested and postfixed in perfusate until sectioning. Before sectioning, a pinhole was made on the right side of the spinal cord to mark laterality. The spinal cords were sectioned transversely at $50 \mu \mathrm{m}$ thickness on a vibratome and placed free floating in PBS.

Sections were washed three times with PBS followed by blocking in 5\% NGS/0.1\% BSA/0.1\% Triton X-100 in PBS for $2 \mathrm{~h}$ at room temperature. After blocking, the sections were incubated in rabbit anti-GFP primary antibody (Invitrogen) overnight at $4^{\circ} \mathrm{C}$. The next day, the sections were washed three times with PBS for 30 min each followed by incubation for $2 \mathrm{~h}$ at room temperature in secondary goat anti-rabbit secondary antibody conjugated to Alexa Fluor 488 (Invitrogen). After washing for three times in PBS at 30 min each, the sections were mounted with 1:1 Citifluor and PBS mounting media on slides and coverslipped. Sections were viewed and imaged on a Zeiss confocal microscope. Cell counting was accomplished by viewing every sixth section (C3-C6) with a Leica fluorescent microscope $(40 \times)$. All cells containing GFP were counted for every sixth section and their numbers were totaled. The estimated cell totals per animal were derived by multiplying the value obtained above by six and then averaging the number per animal for five animals.

\section{Results}

Adult female rats received a left $\mathrm{C} 2$ hemisection by incising from the midline of the spinal cord to the lateral most extent of the spinal cord, just caudal to the $\mathrm{C} 2$ roots. At the same time of hemisection, spinal neurons from C3-C6, the level of the phrenic nucleus, were infected with a Sindbis virus containing ChR2 (1315) fused to GFP (Fig. $1 A$ ). The virus was injected directly into the ventral gray matter of the spinal cord ( 3 injections, $250 \mathrm{nl}$ each, $750 \mathrm{nl}$ total).

Four days after lesion and virus introduction, the C3-C6 spinal cord was exposed again and stimulated with light for physiological characterization and analysis. Before, during and after light stimulation, bilateral diaphragmatic EMG activity was recorded. Successful incorporation of the virus and ChR2-GFP protein expression in spinal neurons were verified and neuroanatomical localization of infected cells was accomplished through GFP reporter detection after physiological recordings.

\section{Expression of $\mathrm{ChR} 2$ in adult spinal neurons}

ChR2-GFP infection and expression was successful in the spinal cord of adult rats. GFP was expressed primarily in ventrally located spinal interneurons and motor neurons (Fig. $1 B$ ). The label was present within the cell soma but also within both the axonal and dendritic compartments. We estimated that there were $\sim 656 \pm 63$ spinal cells infected to express ChR2-GFP per animal. A few astrocytes were also labeled with GFP. Furthermore, after retrograde labeling of PMNs by injecting Dextran Texas Red $(0.4 \%$, five times, $50 \mu \mathrm{l}$ each injection) into the diaphragm muscle, we found that ChR2-GFP was, indeed, expressed in these particular respiratory motor neurons (Fig. $1 C-E$ ). Some motor neurons and interneurons expressing ChR2-GFP had processes that projected toward the midline (Fig. $1 F$ ). In some cases, neurites of labeled interneurons crossed past the central canal and into the contralateral ventral horn (Fig. 1G).

\section{Light-induced stimulation of diaphragmatic EMG activity}

Physiological characterization of rats expressing ChR2-GFP showed that muscular activity after a cervical cord hemisection lesion could be induced in the initially paralyzed hemidiaphragm. Consistent with previous studies (Alilain and Goshgarian, 2008), there was no respiratory related EMG activity present in the hemidiaphragm ipsilateral to the lesion acutely after C2 hemisection and before photostimulation (only EKG activity was present) (Fig. $2 A, B$ ). In our first series of experiments, brief episodic or continuous periods of light stimulation were used $(1 \mathrm{~Hz}$, with each flash of light being $0.5 \mathrm{~s}$ long, total stimulation length was 30-60 s or for continuous stimulation we also used 30-60 s long exposures). Approximately $15 \mathrm{~s}$ after intermittent light stimulation, EMG activity was induced (Fig. $2 \mathrm{~B}$ ). The recorded activity began rhythmically and remained synchronous with respiratory hemidiaphragmatic activity contralateral to the lesion (Fig. $2 \mathrm{~B})$. More remarkably, the activity persisted after photostimulation had ceased and continued for up to $1 \mathrm{~min}$ before dwindling in magnitude and slowly ending (Fig. $2 B, C$ ). This activity was capable of being reproduced multiple times in the same animal after termination of the initial or previous instances of hemidiaphragmatic motor recovery (Fig. 2C). We attempted as many as five repetitions in the same animal, and all were successful. In control animals that received only the GFP construct, activity was absent ipsilateral to the lesion and construct injection before, during, and after photostimulation (Fig. $2 \mathrm{~A}$ ).

In contrast to the results with intermittent photostimulation, continuous episodes of stimulation $(30-60 \mathrm{~s})$ produced hemidiaphragmatic EMG activity in ChR2-GFP infected rats that was tonic, in that the activity was arrhythmic and nonsynchronous with the contralateral, unlesioned side. Furthermore, after termination of the continuous period of stimulation, no kind of diaphragmatic activity, rhythmic or sustained, was detectable on the side ipsilateral to the lesion (data not shown).

In control animals that were infected with ChR2-GFP and received light stimulation but did not receive a hemisection there was also considerable impact on the output of hemidiaphragmatic muscle activity (Fig. 2D,E). Bilateral EMG recordings of the diaphragm showed that during photostimulation, there was a significant increase in tonic EMG activity contralateral to the site 

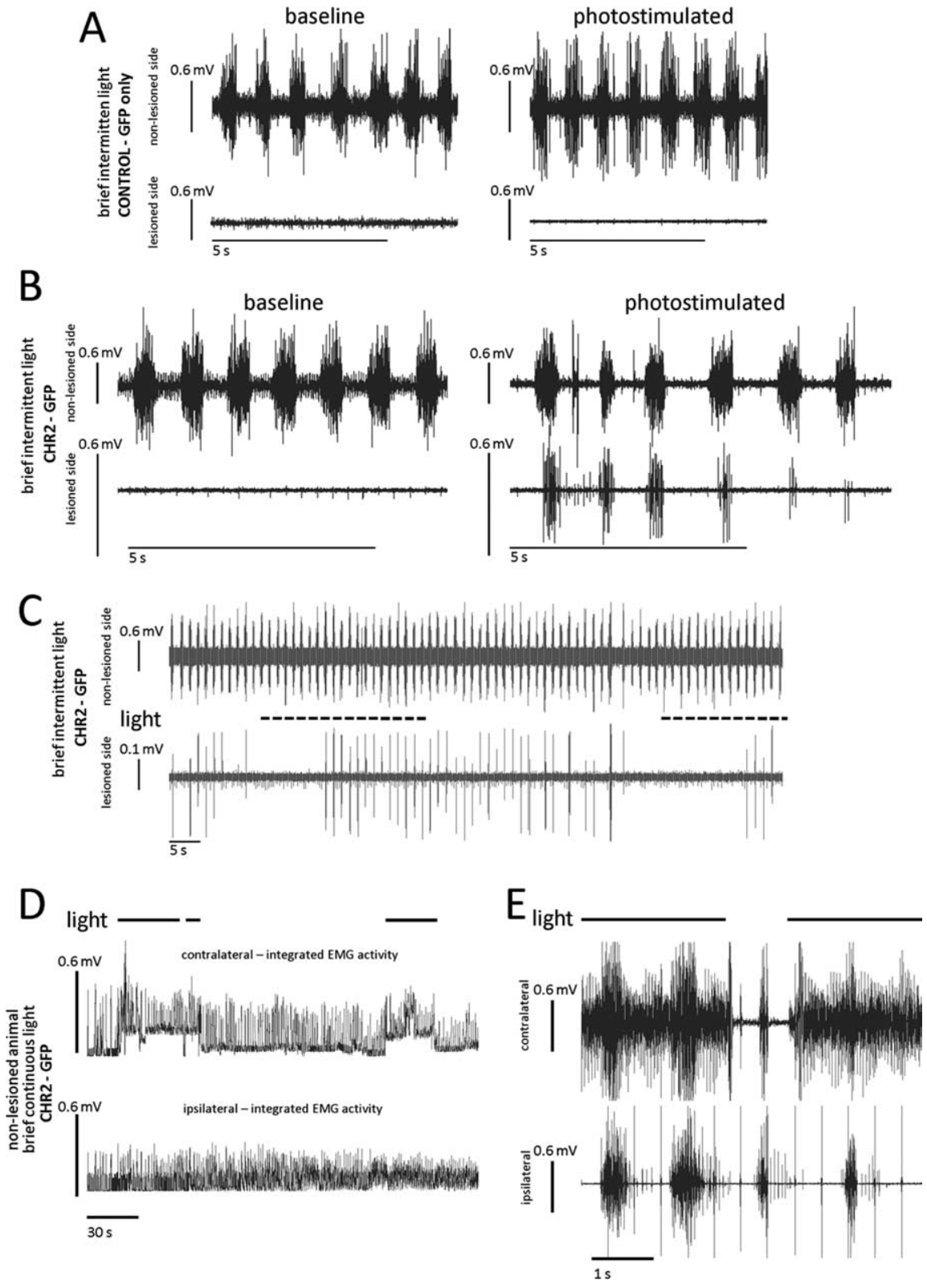

Figure 2. Photostimulation of ChR2-GFP-expressing spinal neurons leads to a return of hemidiaphragmatic EMG activity that can be reinitiated in C2-hemisected animals and can influence the contralateral hemidiaphragm, through midline projecting spinal neurons. $\boldsymbol{A}$, In C2-hemisected animals treated with virus containing only the GFP vector, there is no respiratory activity ipsilateral to the lesion before and after photostimulation (only EKG activity is present). $\boldsymbol{B}$, In (2-hemisected animals that were treated with virus containing the ChR2 and GFP vector, there is no activity before photostimulation. However, after intermittent photostimulation, there is a return of activity that is rhythmic and synchronous with the intact, contralateral side. EMG activity persisted for at least $1 \mathrm{~min}$ after the cessation of photostimulation. After photostimulation induced return of activity, there is a gradual cessation of EMG activity of the hemidiaphragm ipsilateral to the lesion. C, Photostimulation of spinal neurons infected to express ChR2 in C2-hemisected animals can return hemidiaphragmatic activity a number of times in the same animal, including after restored activity has ceased initially. Recovery was repeated up to five times in the same animal. $\boldsymbol{D}, \boldsymbol{E}$, In nonhemisected animals there is a significant increase of hemidiaphragmatic EMG activity contralateral to ChR2-GFP Sindbis virus injection with photostimulation (integrated EMG activity in $\boldsymbol{D}$ and raw EMG activity in $\boldsymbol{E}$ ). There is a slight effect on EMG activity ipsilateral to the injection.

of ChR2-GFP virus injection and infection. Interestingly, in unlesioned animals there were less significant increases of hemidiaphragmatic EMG activity ipsilateral to the expression of ChR2 (Fig. $2 D, E$ ).

Spinal plasticity and adaptation in the spinal cord leading to long-lasting restoration of diaphragmatic EMG activity While further investigating the impact of more intense episodes of intermittent light stimulation after ChR2 expression of spinal neurons, we discovered an unusual, dynamic type of long-term respiratory plasticity that was evident in both $\mathrm{C} 2$ hemisected and unlesioned animals. Compared with the brief, less intense, intermittent stimulation that produced shorter lasting and relatively weak recovery in the first set of experiments; long and patterned intermittent stimulation induced long-lasting recovery. Before photostimulation, no activity was present in the hemidiaphragm ipsilateral to the lesion. However, after and sometimes even during a stimulation protocol that consisted of $5 \mathrm{~min}$ of baseline activity (no light), followed by $5 \mathrm{~min}$ of $0.5 \mathrm{~Hz}$ intermittent light (one second light flash, one second off) for at least three cycles, a trace amount of EMG activity would inevitably appear within the ipsilateral hemidiaphragm. This occurred between 30 and 90 min from the start of the recording session and as late as $1 \mathrm{~h}$ past the last round of photostimulation. The EMG bursting patterns waxed and waned in intensity repetitively in a highly regular pattern, while gradually and dramatically increasing in overall intensity compared with previous periods (Fig. $3 A-C$ ). In addition, bilateral diaphragmatic EMG recordings during these episodes showed an interesting interaction within the phrenic circuitry that controls the two sides of the diaphragm (Fig. $3 B, C$ ). As intense activity on the lesioned side would decrease, EMG activity on the opposite side would increase (Fig. $3 B, C)$. The nonsynchronized increases in activity would oscillate until the phase onsets between the two sides coincided in 30-60 min after the last intermittent light stimulation cycle. The waxing and waning ultimately and slowly disappeared as EMG activity within the once paralyzed hemidiaphragm evolved toward a pattern that closely resembled the nonhemisected side (Fig. 3D,E). This normally patterned breathing lasted for at least $2 \mathrm{~h}$ in the same recording session. After ending the session and waiting $24 \mathrm{~h}$ before beginning another recording session, recovered breathing still persisted but at a lower magnitude (supplemental Fig. 1, available at www.jneurosci.org as supplemental material). Photostimulated control animals not expressing ChR2 did not exhibit this unique pattern of respiratory output (Fig. 3F). Please see supplemental material (available at www.jneurosci.org) for audio and visual recordings. Our analysis showed that although there was no change in frequency of breaths after light stimulation, there were significant increases in peak amplitude and burst duration during inspiratory bursts of the diaphragm bilaterally after photostimulation (Fig. 4A-C). This interesting form of respiratory plasticity was also evident in non-C2 hemisected ani- 


\section{A before long light stimulation B early onset of oscillatory activity}
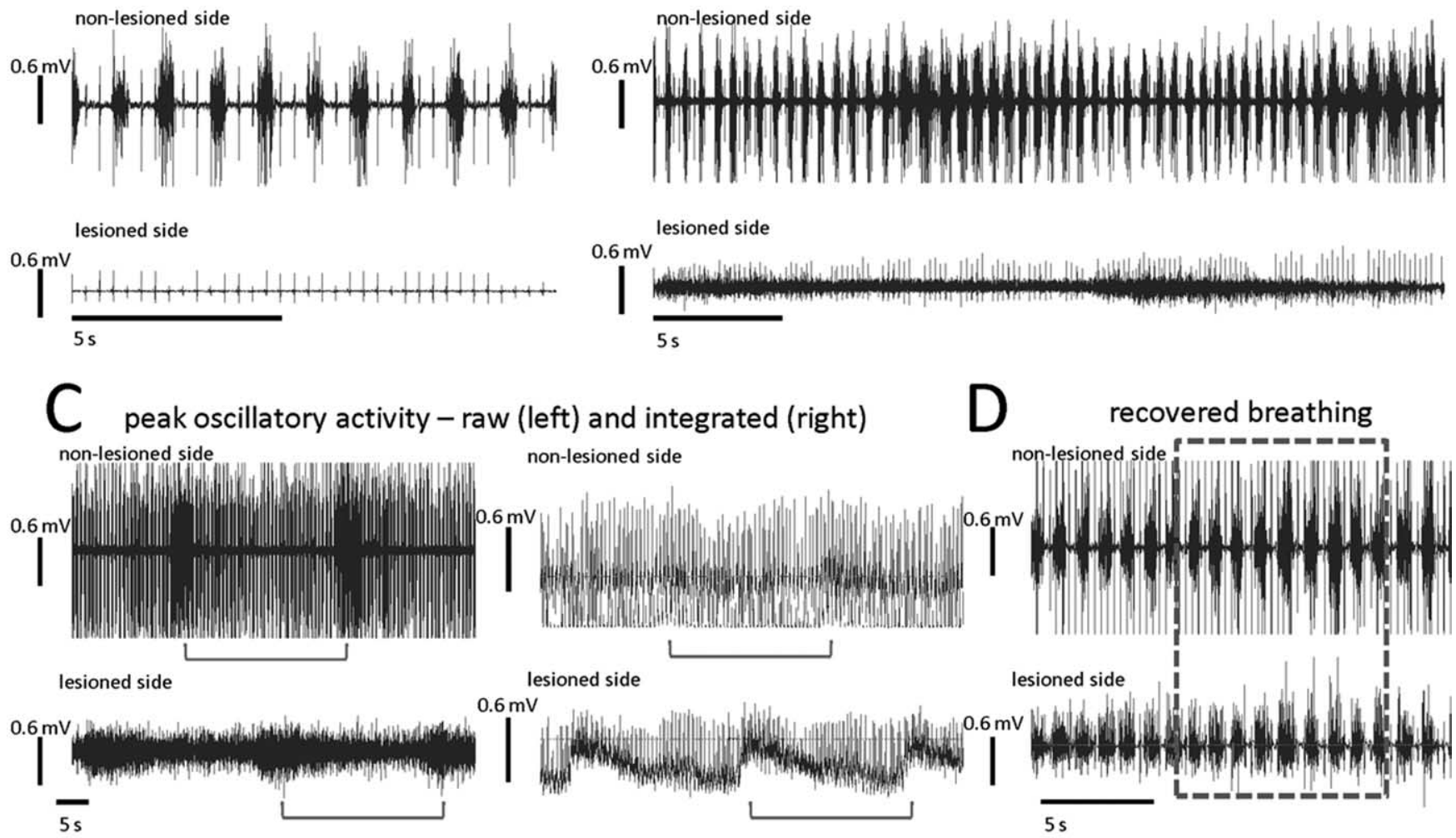

lesioned side
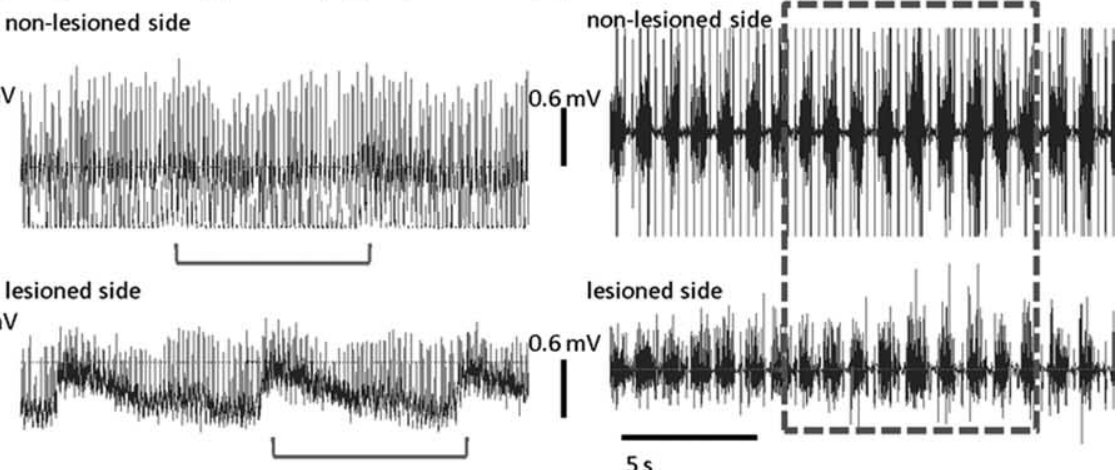

lesioned side

I

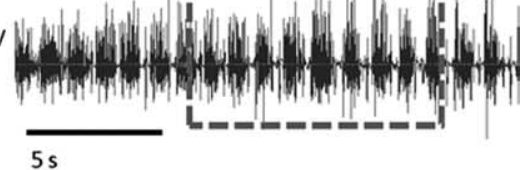

E

$\mathrm{F}$

control - post long light stimulation

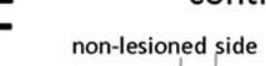
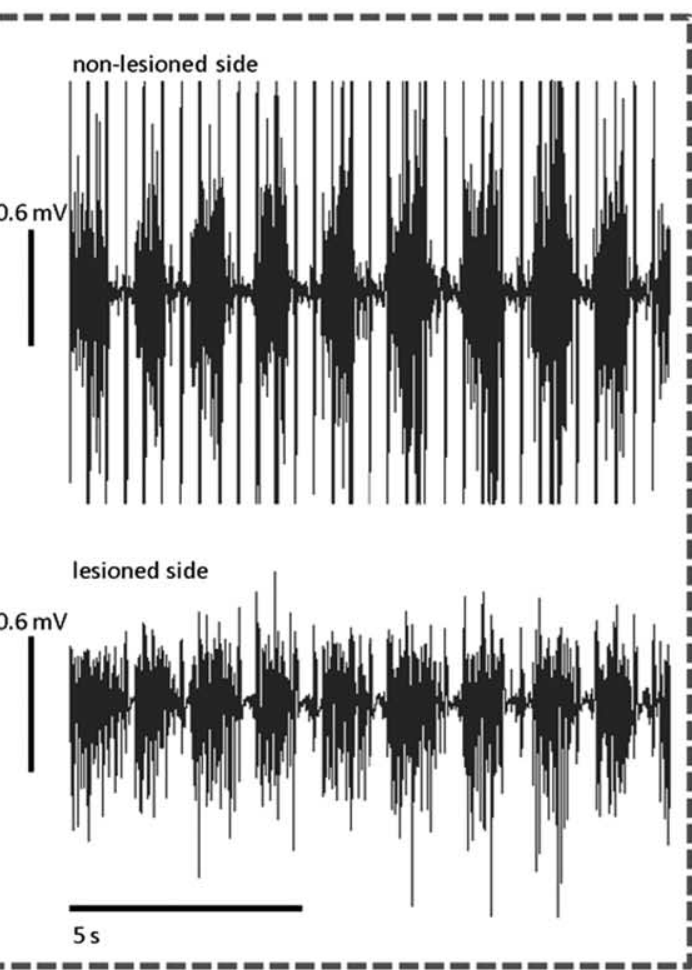

$0.6 \mathrm{mV}$
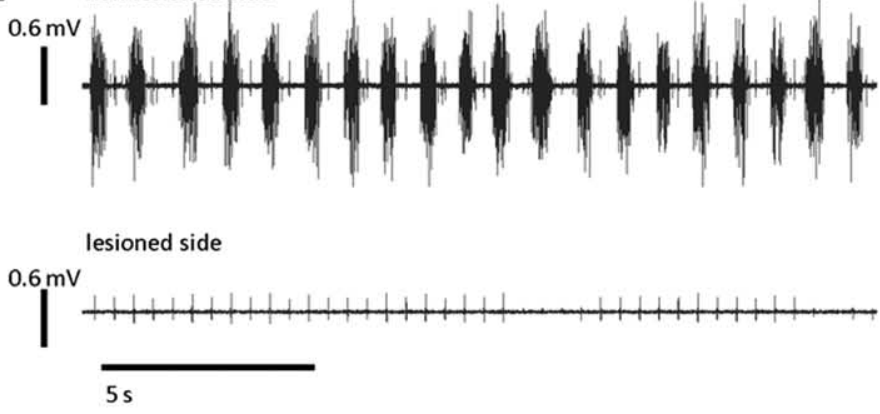

G

unlesioned - post long light stimulation

$0.6 \mathrm{mV}$

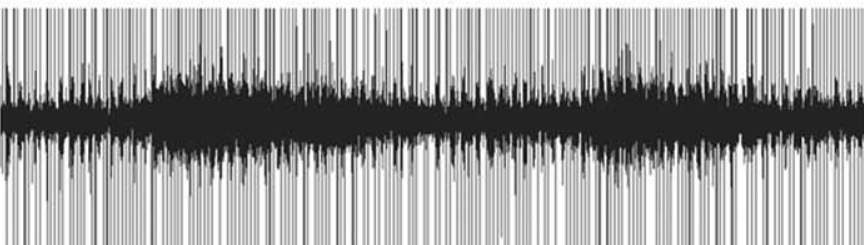

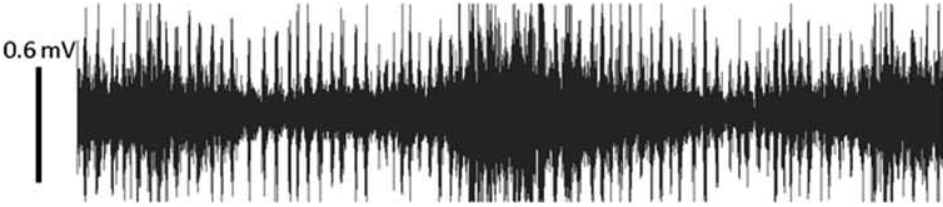

$\overline{5 s}$

Figure 3. Intermittent photostimulation of ChR2-expressing spinal neurons leads to a pattern of EMG hemidiaphragmatic activity that is close to normal in C2-hemisected animals. A, Before photostimulation, there is no EMG activity ipsilateral to the lesion (bottom trace). Contralateral to the lesion, there is rhythmic EMG respiratory activity (top trace). $\boldsymbol{B}$, In the same animal, during the photostimulation protocol of $5 \mathrm{~min}$ off, $5 \mathrm{~min} 0.5 \mathrm{~Hz}$ stimulation, a trace amount of EMG activity begins to develop ipsilateral to the lesion (lower trace). As the EMG activity begins to dwindle, the contralateral, intact side begins to display an increase of EMG activity (upper trace). C, This cycling of high intensity activity that wanes, while the contralateral side increases (Figure legend continues.) 
mals (Fig. 3G). After infection and intermittent light stimulation using the 5 min protocol, oscillating waxing and waning of increasing EMG activity occurred between the two sides of the diaphragm.

NMDA receptor dependence of spinal learning and recovery After application of the noncompetitive NMDA receptor antagonist MK-801 (500 $\mu \mathrm{l}$ of $10 \mu \mathrm{M}$ MK-801 in PBS) to the exposed C3-C6 spinal cord, intermittent photostimulation failed to elicit any kind of change in diaphragmatic EMG activity both ipsilateral as well as contralateral to the ChR2 injection sites in four of six animals (Fig. $4 B, C$ ). In two animals, changes in activity did occur minimally but only contralateral to the lesioned side; and primarily it was an increase of the burst duration of every breath (Fig. 4C). The abolishment of light activated activity by MK-801 was seen in both hemisected and nonlesioned ChR2 animals.

\section{Discussion}

Together, our results suggest that patterned, intermittent photostimulation can potentiate denervated phrenic motor neurons to the usually subthreshold influence of spared pathways, likely the "crossed phrenic pathway," that remains after C2 hemisection (Goshgarian, 2003; Zimmer et al., 2007). Potentiation of PMNs to the crossed phrenic pathway can account for the activity that persisted after cessation of light activation of ChR2, and the rhythmic breathing activity that was observed, because rhythm generation of the respiratory system is primarily supraspinal, although spinal circuits have been identified (Smith et al., 1991; Bellingham, 1999; Feldman and Del Negro, 2006).

\section{Both adult spinal motorneurons and interneurons can express ChR2 and can influence the contralateral side}

Our data also showed that there were changes in diaphragmatic EMG activity contralateral to the site of ChR2 expression. Interestingly, there appears to be a subset of neurons, possibly ChR2expressing interneurons or, less likely, motor neurons that can influence contralateral phrenic motor neurons after activation with photostimulation. After further examination of GFP expression in $\mathrm{C} 4$ spinal cord cross sections, both interneurons and motor neurons were capable of projecting neurites toward the midline. In fact, some interneuronal processes crossed the midline within the ventral white commissure to the contralateral side. Recent anatomical studies have suggested that interneurons may play a significant role in mediating crossed phrenic activity (Lane et al., 2008). Our physiological data provide strong support for the functional influence of contralaterally projecting cells on phrenic motor circuitry.

These sets of experiments further suggest a sophisticated level of connectivity and circuitry related to respiration between the two sides of the spinal cord that has not been observed before in the rat. In addition to the functional bilaterality of interconnections at the level of the phrenic motor pool, the oscillating patterns of EMG activity that slowly build toward normal levels and

$\leftarrow$

(Figure legend continued.) activity, continues with each period of high intensity activity being slightly more than the last ( $\boldsymbol{C}$ compared with $\boldsymbol{B}$ ), and this is after the last round of photostimulation. The left two traces are of the raw EMG signal, and the right is of the same time point but integrated and rectified. Brackets under traces indicate periods between onsets of increased diaphragmatic EMG activity. $\boldsymbol{D}, \boldsymbol{E}$, Eventually EMG activity becomes closer to normal patterned respiratory EMG activity. $\boldsymbol{E}$, Inset of $\boldsymbol{D}$. $\boldsymbol{F}, A$ trace of control-treated animal after photostimulation. $\boldsymbol{G}, A$ representative trace of the waxing and waning exhibited by non-C2-hemisected animals that expressed ChR2 and were photostimulated. Top trace is of the injected side.

\section{A \\ Frequency of Breaths}
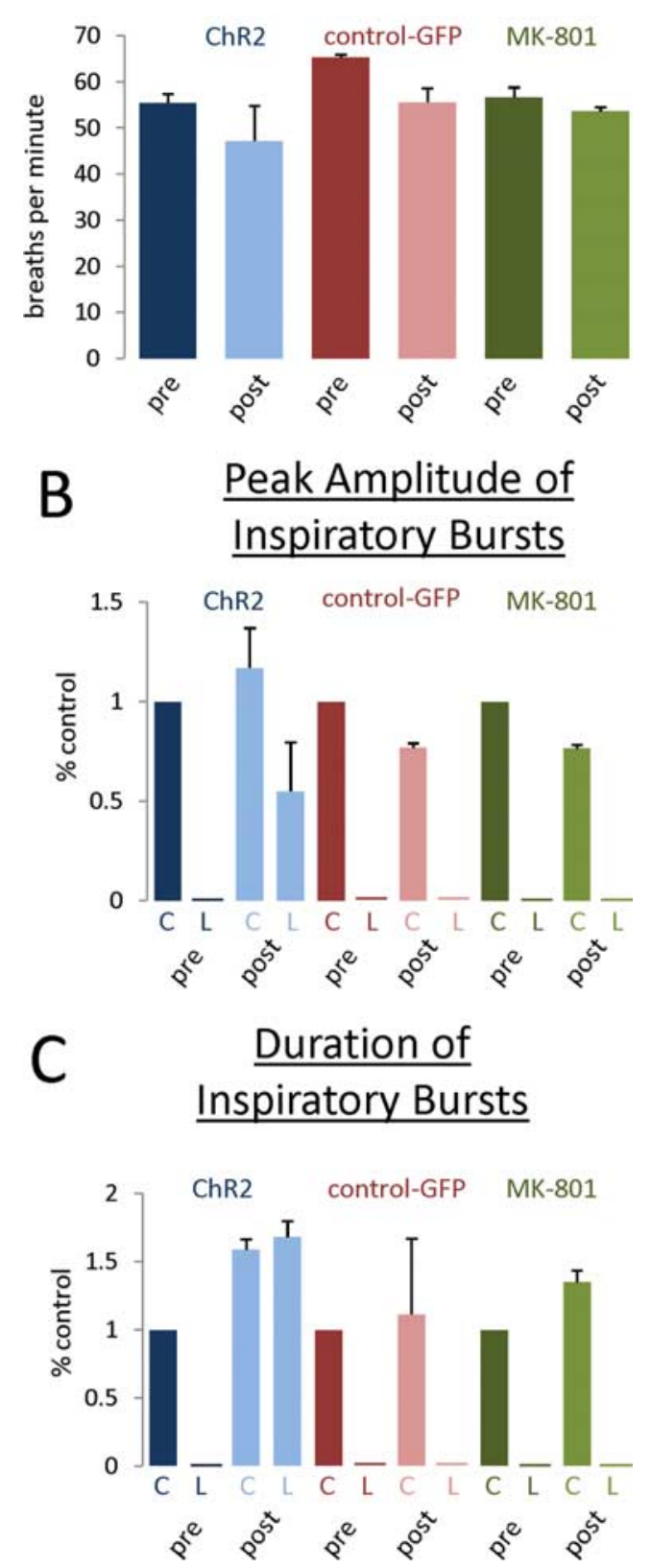

Figure 4. Induction of respiratory plasticity and recovery of hemidiaphragmatic EMG activity results in increases of average peak amplitude and duration of inspiratory bursts after recovery of breathing which is NMDA receptor dependent. $\boldsymbol{A}$, There was no change in the frequency of breaths before and after stimulation in ChR2-expressing animals, GFP-expressing animals, and MK-801treated animals. $\boldsymbol{B}$, After photostimulation, there was an increase of peak EMG amplitude during inspiratory bursts bilaterally in photostimulated ChR2 animals (blue bars). After blockade with MK801, this increase was abolished (green bars) and brought back to control levels (red bars). C, After photostimulation, there was an increase in the duration of EMG inspiratory bursts bilaterally in photostimulated ChR2 animals (blue bars). After blockade with MK-801, the increase in duration was attenuated (green bars) and brought back to control levels (red bars). Measurements of postphotostimulated animals were made where normal patterned breathing had occurred, i.e., postoscillatory phasic activity. C, Control, nonlesioned side; L, lesioned side.

synchrony resulting in recovery of normal patterned breathing, suggests the idea of synaptic strengthening or plasticity within spinal respiratory circuitry which can adapt and learn so that functional activity that is normal in pattern can emerge. It is also 
possible that our long light stimulation protocol has revealed a dormant, spinal respiratory circuit that is similar to a central pattern generator (CPG) whose activation leads to the alternating firing rhythm that develops between the two sides of the diaphragm (Whelan et al., 2000).

Our observation that there is an increase in background or tonic activity during light stimulation suggests that a variety of spinal interneurons or possibly even glia expressing ChR2 may have inputs to the primary spinal circuitry mediating respiration. Using more specific neuronal promoters, the precise role of each cell type in the restoration of respiratory activity can be dissected. Furthermore, because there is a delay and slow augmentation in respiratory related EMG activity in animals with both brief and long light stimulation, it is possible that more widespread alterations in circuit activation via recruitment of respiratory associated spinal neurons not expressing ChR2 is required for the recovery process. It is conceivable that the episodes that initially emerge, especially after long light exposure, parallel the kinds of events that occur during the phenomenon of kindling, in which patterned, low-intensity electrical stimulation can spread to nearby circuits, leading to progressive amounts of CNS activity even after stimulation has ceased (Bertram, 2007). Interestingly, kindling, which, in turn, can lead to the induction of epileptiform activity, is also partially glutamate and NMDA receptor activation associated (Martin et al., 1992, Kraus et al., 1994, Morimoto et al., 2004). However, in the lesioned spinal cord, where there is a dearth of activity, light-induced "kindling" and the onset of seizure-like activity somehow become regulated in a beneficial way. This is probably because of the continuing influence of the normal respiratory rhythm being generated from the brainstem as well as the presence of relatively intact seizure dampening mechanisms within the spinal cord, including the effects of astrocytes and inhibitory interneurons (Trotter et al., 2006 and Eid et al., 2008).

\section{A model of ChR2 activation that can lead to long-lasting recovery of muscle activity after spinal cord injury}

One component that plays an important role in activitydependent synaptic plasticity, learning, and adaptation in the CNS is the glutamatergic NMDA receptor. Our observation that the NMDA receptor antagonist MK-801 eliminated cycling of increasing diaphragmatic EMG activity after photostimulation begins to suggest a mechanism underlying this form or respiratory plasticity, recovery, and synaptic strengthening (Fig. 5). Because the NMDA receptor is a voltage-gated ionotropic glutamate receptor, the depolarization caused by photostimulation of ChR2 could result in release of the $\mathrm{Mg}^{2+}$ ion blocking the channel (Fig. 5) (Moriyoshi et al., 1991). Once released of this block, $\mathrm{Ca}^{2+}$ influx can occur and a series of signaling cascades can begin leading to activation of the protein kinase C/RAF/MAP kinase sequence, and/or the SRC/Grb2/Sos sequence. In turn, both of these pathways can lead to initiation of ERK, increased protein synthesis, and/or immediate early gene translation (Rusanescu et al., 1995; Waltereit and Weller, 2003; Sun and June Liu, 2007). $\mathrm{Ca}^{2+}$ can also enter directly via ChR2 during light stimulation adding to these processes. Regardless of the downstream molecular cascade that might be involved, the NMDA receptor has been identified as a primary mediator of learning and long-term potentiation (LTP) in the hippocampus, in the induction of another mechanism of respiratory plasticity known as long-term facilitation (LTF), and in the spontaneous respiratory recovery observed after C2 hemisection (Seeburg et al., 1995; Tang et al., 1999; McGuire et al., 2005; Alilain and Goshgarian, 2008). During the initiation of LTP and LTF, these forms of plasticity require intermittent stimulation and the plasticity we have uncovered may be analogous to or use the same cellular machinery as these events (Seeburg et al., 1995; Tang et al., 1999; McGuire et al., 2005).

From these experiments we can begin to hypothesize that there is a subthreshold level of patterned glutamate being released from spared pathways, because the NMDA receptor also requires glutamate binding to be activated along with membrane depolarization (Fig. 5). This sparse glutamatergic transmission may be potentiated on either phrenic motor neurons, interneurons or both through increased receptor presence on the postsynaptic membrane, phosphorylation of present receptors, or some totally new mechanism (Fig. 5) (Isaac et al., 1995; Rumpel et al., 1998; Esteban, 2003). Other voltage-gated $\mathrm{Ca}^{2+}$ channels, such as the $\mathrm{L} / \mathrm{N} / \mathrm{P} / \mathrm{Q} / \mathrm{T}$ types, may also play a role in our observations and account for the limited response we saw in two MK-801-treated animals (Fig. 4 B) (Dolphin, 2006). Finally if, indeed, this plastic- 
ity is $\mathrm{Ca}^{2+}$ dependent, the fine tuning of EMG activity may be mediated through activated $\mathrm{Ca}^{2+} \mathrm{SK}$ channels which accompany NMDA receptor activation and LTP (Stocker, 2004). In the end, we will need to understand how the molecular machinery that is driven by intermittent depolarization and/or activation of phrenic motor neurons or spinal respiratory interneurons by light leads to an activity-dependent plasticity and circuit adaptation that is slow to emerge but becomes robust then regularly patterned and long lasting.

In conclusion, we have demonstrated that activation of C3-C6 spinal neurons, including denervated phrenic motor neurons or interneurons, some with contralateral projections, through stimulation of the ChR2 protein can restore repeatedly, diaphragmatic muscle activity that is rhythmic and persistent even after the cessation of light. This is the first time that this emerging technology has been successfully used after traumatic CNS injury to restore activity. Our data suggests that after debilitating lesions of the CNS, which leave CNS neurons denervated and quiescent, incorporation of the algal protein ChR2 (as well as the hyperpolarizing off-switches, vertebrate rhodopsin 4 or halorhodopsin) and subsequent photostimulation of infected neurons is a possible alternative to restore and control neuronal activity, possibly through potentiation of denervated target neurons and supersensitivity to spared axonal inputs (Li et al., 2005; Teng et al., 2006, Herlitze and Landmesser, 2007; Zhang et al., 2007a,b). In the case of SCI, which can leave entire spinal motor neuron pools with zero or only minimal amounts of supraspinal input, this exciting and potential therapy is one that should be further explored and studied. With the perfection of an in vivo light source, it can be envisioned that more chronic manipulation and stimulation of spinal neurons or neuronal circuits, including spinal central pattern generators through light, can lead to recovery of lost function after SCI including bowel and bladder function and possibly walking to improve the quality of life of SCI patients (Lynch and Frizelle, 2006; Potter, 2006; Yakovenko et al., 2007; Fowler et al., 2008).

\section{References}

Alilain WJ, Goshgarian HG (2008) Glutamate receptor plasticity and activity-regulated cytoskeletal associated protein regulation in the phrenic motor nucleus may mediate spontaneous recovery of the hemidiaphragm following chronic cervical spinal cord injury. Exp Neurol 212:348-357.

Arenkiel BR, Peca J, Davison IG, Feliciano C, Deisseroth K, Augustine GJ, Ehlers MD, Feng G (2007) In vivo light-induced activation of neural circuitry in transgenic mice expressing channelrhodopsin-2. Neuron 54:205-218.

Bellingham MC (1999) Synaptic inhibition of cat phrenic motoneurons by internal intercostal nerve stimulation. J Neurophysiol 82:1224-1232.

Bertram E (2007) The relevance of kindling for human epilepsy. Epilepsia 48 [Suppl 2]:65-74.

Boyden ES, Zhang F, Bamberg E, Nagel G, Deisseroth K (2005) Millisecondtimescale genetically targeted optical control of neural activity. Nat Neurosci 8:1263-1268.

Dolphin AC (2006) A short history of voltage-gated calcium channels. Br J Pharmacol 147 [Suppl 1]:S56-S62.

Eid T, Williamson A, Lee TS, Petroff OA, de Laneraolle NC (2008) Glutamate and astrocytes-key players in human mesial temporal lobe epilepsy? Epilepsia 49 [Suppl 2]:42-52.

Esteban JA (2003) AMPA receptor trafficking: a roadmap for synaptic plasticity. Mol Interv 3:375-385.

Feldman JL, Del Negro CA (2006) Looking for inspiration: new perspectives on respiratory rhythm. Nat Rev Neurosci 7:232-242.

Fowler CJ, Griffiths D, de Groat WC (2008) The neural control of micturition. Nat Rev Neurosci 9:453-466.

Fuller DD, Johnson SM, Olson EB Jr, Mitchell GS (2003) Synaptic pathways to phrenic motoneurons are enhanced by chronic intermittent hypoxia after cervical spinal cord injury. J Neurosci 23:2993-3000.

Golder FJ, Mitchell GS (2005) Spinal synaptic enhancement with acute intermittent hypoxia improves respiratory function after chronic cervical spinal cord injury. J Neurosci 25:2925-2932.

Goshgarian HG (2003) The crossed phrenic phenomenon: a model for plasticity in the respiratory pathways following spinal cord injury. J Appl Physiol 94:795-810.

Herlitze S, Landmesser LT (2007) New optical tools for controlling neuronal activity. Curr Opin Neurobiol 17:87-94.

Houle JD, Tom VJ, Mayes D, Wagoner G, Phillips N, Silver J (2006) Combining an autologous peripheral nervous system "bridge" and matrix modification by chondroitinase allows robust, functional regeneration beyond a hemisection lesion of the adult rat spinal cord. J Neurosci 26:7405-7415.

Huber D, Petreanu L, Ghitani N, Ranade S, Hromádka T, Mainen Z, Svoboda K (2008) Sparse optical microstimulation in barrel cortex drives learned behaviour in freely moving mice. Nature 451:61-64.

Isaac JT, Nicoll RA, Malenka RC (1995) Evidence for silent synapses: implications for the expression of LTP. Neuron 15:427-434.

Kim J, Dittgen T, Nimmerjahn A, Waters J, Pawlak V, Helmchen F, Schlesinger S, Seeburg PH, Osten P (2004) Sindbis vector SINrep(nsP2S726): a tool for rapid heterologous expression with attenuated cytotoxicity in neurons. J Neurosci Methods 133:81-90.

Kraus JE, Yeh GC, Bonhaus DW, Nadler JV, McNamara JO (1994) Kindling induces the long-lasting expression of a novel population of NMDA receptors in hippocampal region CA3. J Neurosci 14:4196-4205.

Lagali PS, Balya D, Awatramani GB, Münch TA, Kim DS, Busskamp V, Cepko CL, Roska B (2008) Light-activated channels targeted to ON bipolar cells restore visual function in retinal degeneration. Nat Neurosci 11:667-675.

Lane MA, Fuller DD, White TE, Reier PJ (2008) Respiratory neuroplasticity and cervical spinal cord injury: translational perspectives. Trends Neurosci 31:538-547.

Li X, Gutierrez DV, Hanson MG, Han J, Mark MD, Chiel H, Hegemann P, Landmesser LT, Herlitze S (2005) Fast noninvasive activation and inhibition of neural and network activity by vertebrate rhodopsin and green algae channelrhodopsin. Proc Natl Acad Sci U S A 102:17816-17821.

Lynch AC, Frizelle FA (2006) Colorectal motility and defecation after spinal cord injury in humans. Prog Brain Res 152:335-343.

Martin D, McNamara JO, Nadler JV (1992) Kindling enhances sensitivity of CA3 hippocampal pyramidal cells to NMDA. J Neurosci 12:1928-1935.

McGuire M, Zhang Y, White DP, Ling L (2005) Phrenic long-term facilitation requires NMDA receptors in the phrenic motonucleus in rats. J Physiol 567:599-611.

Morimoto K, Fahnestock M, Racine RJ (2004) Kindling and status epilepticus models of epilepsy: rewiring the brain. Prog Neurobiol 73:1-60.

Moriyoshi K, Masu M, Ishii T, Shigemoto R, Mizuno N, Nakanishi S (1991) Molecular cloning and characterization of the rat NMDA receptor. Nature 354:31-37.

Nantwi KD, El Bohy A, Schrimsher GW, Reier PJ, Goshgarian HG (1999) Spontaneous functional recovery in paralyzed hemidiaphragm following upper cervical spinal cord injury in adult rats. Neurorehabil Neural Repair 13:225-234.

National Spinal Cord Injury Statistical Center (2006) Spinal cord injury: facts and figures at a glance. J Spinal Cord Med 29:89-90.

Porter WT (1895) The path of the respiratory impulse from the bulb to the phrenic nuclei. J Physiol 17:455-485.

Potter PJ (2006) Disordered control of the urinary bladder after human spinal cord injury: what are the problems? Prog Brain Res 152:51-57.

Rumpel S, Hatt H, Gottmann K (1998) Silent synapses in the developing rat visual cortex: evidence for postsynaptic expression of synaptic plasticity. J Neurosci 18:8863-8874.

Rusanescu G, Qi H, Thomas SM, Brugge JS, Halegoua S (1995) Calcium influx induces neurite growth through a Src-Ras signaling cassette. Neuron 15:1415-1425.

Seeburg PH, Burnashev N, Köhr G, Kuner T, Sprengel R, Monyer H (1995) The NMDA receptor channel: molecular design of a coincidence detector. Recent Prog Horm Res 50:19-34.

Smith JC, Ellenberger HH, Ballanyi K, Richter DW, Feldman JL (1991) PreBötzinger complex: a brainstem region that may generate respiratory rhythm in mammals. Science 254:726-729. 
Stocker M (2004) Ca(2+)-activated K+ channels: molecular determinants and function of the SK family. Nat Rev Neurosci 5:758-770.

Sun L, June Liu S (2007) Activation of extrasynaptic NMDA receptors induces a PKC-dependent switch in AMPA receptor subtypes in mouse cerebellar stellate cells. J Physiol 583:537-553.

Tang YP, Shimizu E, Dube GR, Rampon C, Kerchner GA, Zhuo M, Liu G, Tsien JZ (1999) Genetic enhancement of learning and memory in mice. Nature 401:63-69.

Teng YD, Liao WL, Choi H, Konya D, Sabharwal S, Langer R, Sidman RL, Snyder EY, Frontera WR (2006) Physical activity-mediated functional recovery after spinal cord injury: potential roles of neural stem cells. Regen Med 1:763-776.

Trotter SA, Kapur J, Anzivino MJ, Lee KS (2006) GABAergic synaptic inhibition is reduced before seizure onset in a genetic model of cortical malformation. J Neurosci 26:10756-10767.

Waltereit R, Weller M (2003) Signaling from cAMP/PKA to MAPK and synaptic plasticity. Mol Neurobiol 27:99-106.

Whelan P, Bonnot A, O’Donovan MJ (2000) Properties of rhythmic activity generated by the isolated spinal cord of the neonatal mouse. J Neurophysiol 84:2821-2833.
Yakovenko S, Kowalczewski J, Prochazka A (2007) Intraspinal stimulation caudal to spinal cord transections in rats. Testing the propriospinal hypothesis. J Neurophysiol 97:2570-2574.

Zhang F, Wang LP, Boyden ES, Deisseroth K (2006) Channelrhodopsin-2 and optical control of excitable cells. Nature Methods 3:785-792.

Zhang F, Aravanis AM, Adamantidis A, de Lecea L, Deisseroth K (2007a) Circuit-breakers: optical technologies for probing neural signals and systems. Nat Rev Neurosci 8:577-581.

Zhang F, Wang LP, Brauner M, Liewald JF, Kay K, Watzke N, Wood PG, Bamberg E, Nagel G, Gottschalk A, Deisseroth K (2007b) Multimodal fast optical interrogation of neural circuitry. Nature 446:633-669.

Zhang YP, Oertner TG (2007) Optical induction of synaptic plasticity using a light-sensitive channel. Nat Methods 4:139-141.

Zhang YP, Holbro N, Oertner TG (2008) Optical induction of plasticity at single synapses reveals input-specific accumulation of alphaCaMKII. Proc Natl Acad Sci U S A 105:12039-12044.

Zimmer MB, Nantwi K, Goshgarian HG (2007) Effect of spinal cord injury on the respiratory system: basic research and current clinical treatment options. J Spinal Cord Med 30:319-330. 